\title{
Comparison Between Neuromuscular Electrical Stimulation to Abdominal and Back Muscles on Postural Balance in Post-stroke Hemiplegic Patients
}

\author{
Mingeun Park, MD, Hyun Seok, MD, PhD, Sang-Hyun Kim, MD, PhD, \\ Kyudong Noh, MD, Seung Yeol Lee, MD
}

Department of Physical Medicine and Rehabilitation, Soonchunhyang University Bucheon Hospital, Soonchunhyang University College of Medicine, Bucheon, Korea

\begin{abstract}
Objective To compare the effects of neuromuscular electrical stimulation (NMES) to abdominal muscles and back muscles on postural balance in post-stroke hemiplegic patients.

Methods Thirty post-stroke hemiplegic patients were prospectively enrolled and randomly assigned to one of the three groups: core muscle-strengthening exercise (CME) with NMES to abdominal muscles (group A), CME with NMES to back muscles (group B), and CME alone (group C). All subjects underwent their targeted interventions for 30 minutes each day, 5 days per week for 3 weeks under a conventional stroke rehabilitation program. Subjects were evaluated using Korean version of Berg Balance Scale (K-BBS), Trunk Impairment Scale (TIS), Korean version of Modified Barthel Index (K-MBI), Weight Distribution Index (WDI), and Stability Index (SI) just before and 3 weeks after intervention. Results Changes in K-BBS $(\mathrm{p}<0.05)$ and TIS $(\mathrm{p}<0.05)$ were significantly higher in group A $(18.5 \pm 8.10,6.6 \pm 1.90)$ and group B $(19.9 \pm 5.44,7.0 \pm 2.26)$ than in group C $(8.4 \pm 4.14,3.1 \pm 0.99)$. However, K-MBI, WDI, and SI failed to show any significant difference. No significant difference in all outcomes was observed between groups A and B.

Conclusion The effect of NMES to the abdominal muscles was similar to the effect on back muscles in terms of postural balance. This finding indicated that the NMES to the abdominal muscles may be an alternative for post-stroke hemiplegic patients contraindicated for NMES to the back muscles. Additional studies investigating the effects of NMES on abdominal and back muscles are needed.
\end{abstract}

Keywords Stroke, Abdominal muscles, Electrical stimulation, Postural balance

Received March 19, 2018; Accepted June 18, 2018

Corresponding author: Seung Yeol Lee

Department of Physical Medicine and Rehabilitation, Soonchunhyang University Hospital, 170 Jomaru-ro, Wonmi-gu, Bucheon 14584, Korea. Tel: +8232-621-5057, Fax: +82-32-621-6148, E-mail: shouletz@gmail.com

ORCID: Mingeun Park (https://orcid.org/0000-0002-2420-3473); Hyun Seok (https://orcid.org/0000-0001-7266-6045); Sang-Hyun Kim (https://orcid. org/0000-0003-4475-5571); Kyudong Noh (https://orcid.org/0000-0002-7207-6317); Seung Yeol Lee (https://orcid.org/0000-0003-1571-9408).

(c) This is an open-access article distributed under the terms of the Creative Commons Attribution Non-Commercial License (http://creativecommons.org/ licenses/by-nc/4.0) which permits unrestricted noncommercial use, distribution, and reproduction in any medium, provided the original work is properly cited. Copyright $\odot 2018$ by Korean Academy of Rehabilitation Medicine 


\section{INTRODUCTION}

Core muscle acts as the center of almost all kinetic chains. A stable and strong core muscle contributes to the effective use of limbs. Core muscle stability refers to the ability of the lumbopelvic-hip complex in preventing buckling of the vertebrae and facilitating the return of unstable vertebrae to a normal position [1-3].

Stroke can cause a variety of impairments, such as muscle weakness and decline of balance function, and increase the risk of falls and decrease the ability to perform activities of daily living (ADL) [4-6].

Several studies have shown that core muscle exercises in stroke patients improved trunk muscle strength and postural stability [7-9]. Kim et al. [10] reported that neuromuscular electrical stimulation (NMES) improved trunk stability in acute and subacute stroke patients. Ko et al. [6] reported that a combination of core muscle strengthening exercise (CME) and NMES to back muscles improved patients' postural stability more than either therapy alone. Fujita et al. [9] showed that abdominal muscle strengthening exercise improves the performance of ADL. However, few studies evaluated the effects of NMES to abdominal muscles in maintaining postural balance.

The aim of this study was to compare the effects of NMES to abdominal and back muscles on postural balance in post-stroke hemiplegic patients.

\section{MATERIALS AND METHODS}

\section{Participants}

The inclusion criteria were as follows: (1) subjects who

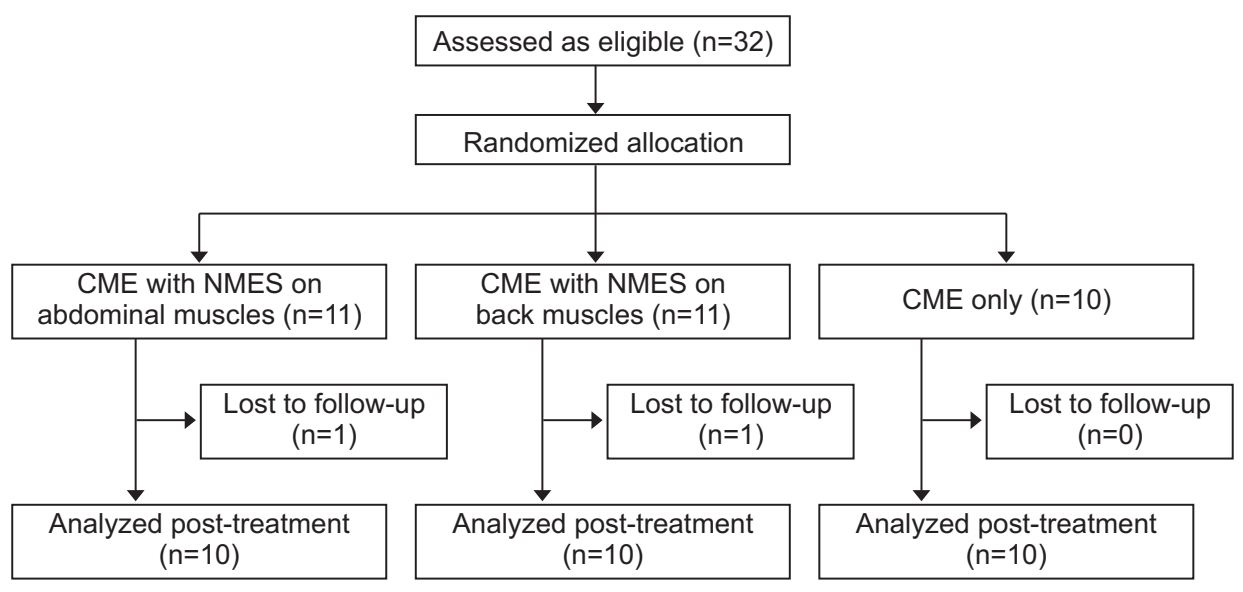

were diagnosed with stroke, indicated by magnetic resonance imaging or computed tomography, and an onset time of less than 6 months; (2) subjects who had no previous history of stroke; and (3) subjects who maintained static sitting balance for more than 5 minutes. The exclusion criteria were as follows: (1) subjects with vestibular, orthopedic, medical or other neurologic conditions affecting postural stability; (2) subjects who were uncooperative because of severe aphasia or cognitive impairment; (3) subjects with uncontrolled medical conditions; (4) subjects with neglect syndromes; and (5) subjects with implanted pacemakers of defibrillators.

The sample size was calculated using the G*Power version 3.1.9.2 (http://www.gpower.hhu.de/). The power was set at 0.80 , with an alpha of 0.05 , and effect size 0.70 . Assuming an attrition rate of $20 \%$, an estimated total sample size of 30 (10 per each group) was needed.

A total of 32 subjects were randomly assigned to three groups by selecting the card with the group number in the invisible box: CME with NMES on abdominal muscles (group $\mathrm{A}, \mathrm{n}=11$ ), CME with NMES on back muscles (group $\mathrm{B}, \mathrm{n}=11$ ), and CME alone (group $\mathrm{C}, \mathrm{n}=10$ ). However, one subject from group A dropped out due to aspiration pneumonia, and a follow-up loss in group B occurred due to early discharge before the study completion. Thus, 30 subjects completed the study and were analyzed (Fig. $1)$.

All participants were informed of the procedures, and objectives of the study. The study protocol was approved by the Institutional Review Board of Soonchunhyang University Bucheon Hospital (No. SCHBC2017-09-003).

Fig. 1. Flow diagram of the study. CME, core muscle strengthening exercise; NMES, neuromuscular electrical stimulation. 


\section{Intervention}

All subjects underwent conventional stroke rehabilitation program consisting of physical and occupational therapy including range of motion exercises, aerobic exercise, strengthening exercise, sitting and standing balance training using mirror or balance board, basic and instrumental ADL training, progressive gait and functional ambulation training. Cognitive or speech therapy was added as needed.

In addition to conventional rehabilitation, all three groups underwent their targeted interventions for 30 minutes each day, 5 days per week for 3 weeks. The CME program and the location of NMES attachment in abdominal and back muscles were similar to those reported in recently published trials $[6,11]$.

NMES (Microstim; SejinMT Co. Ltd., Seoul, Korea) was administered at 30-70 mA intensity, $250 \mathrm{~ms}$ pulse width, and $35 \mathrm{~Hz}$ frequency for 10 seconds followed by a pause of 12 seconds to the abdominal muscles (group A) and the back muscles (group B) during the CME. The intensity of stimulation was set to the maximum amount at which patients felt muscle contractions without pain sensation or fatigue. In group A, electrodes were attached 1 $\mathrm{cm}$ superior to the iliac crest along the mid-axillary line, and $2 \mathrm{~cm}$ superior and $2 \mathrm{~cm}$ medial to the anterior superior iliac spine, bilaterally (Fig. 2). In group B, electrodes were attached to the bilateral thoracic erector spinae ( 5

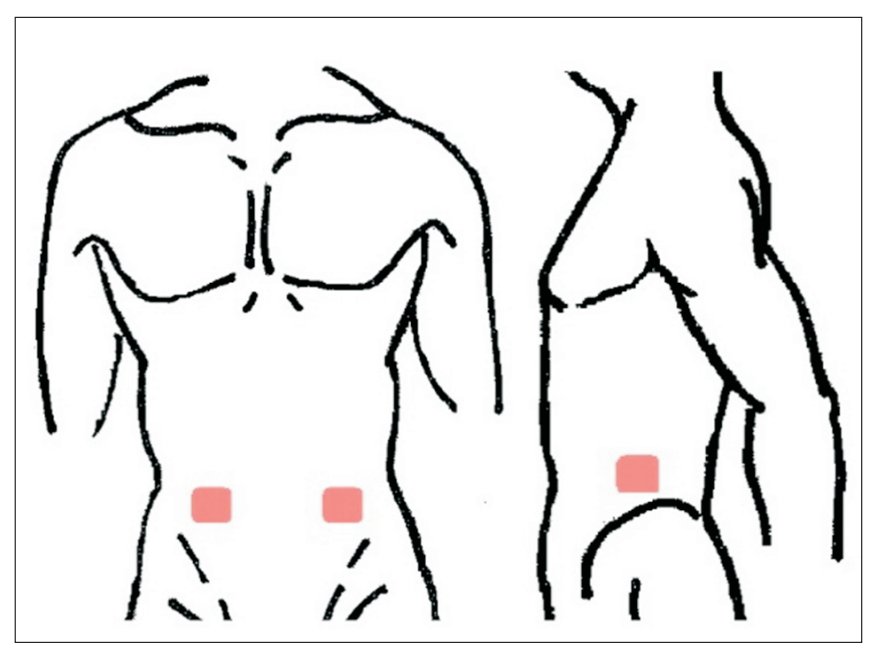

Fig. 2. Surface electrodes were attached to abdominal muscles bilaterally at 4 sites. Two electrodes were attached $1 \mathrm{~cm}$ superior to the iliac crest along the midaxillary line, and the remaining electrodes $2 \mathrm{~cm}$ superior and $2 \mathrm{~cm}$ medial to the anterior superior iliac spine. cm lateral to the T6 spinous process) and lumbar erector spinae ( $2 \mathrm{~cm}$ lateral to the L5 spinous process) (Fig. 3). All electrodes measured $5 \times 5 \mathrm{~cm}$ in size.

The CME program consisted of the following exercises, listed by position: (1) supine position-bridge exercise, segmental rotation, dead bug exercise; (2) prone position-plank exercise, belly blaster, bird dog exercise; and (3) lateral position-side plank exercise, side bridge exercise.

Exercise intensity was increased gradually according to the patients' individual levels of tolerance.

\section{Outcome measures}

All outcome measurements were assessed just before and 3 weeks after intervention.

Scores on the Korean version of Berg Balance Scale (KBBS; score range 0-56) and Trunk Impairment Scale (TIS; score range 0-23) for postural balance, and Korean version of Modified Barthel Index (K-MBI; score range 0-100) for ADL were used as the primary outcome to confirm the effectiveness of the intervention and compared with previous studies.

For secondary outcome, Weight Distribution Index (WDI) and Stability Index (SI) were evaluated using Tetrax (Sunlight Medical Ltd., Israel) (Fig. 4). WDI indicates a significant discrepancy from equal weight distribution. SI suggests general stability and ability to compensate for

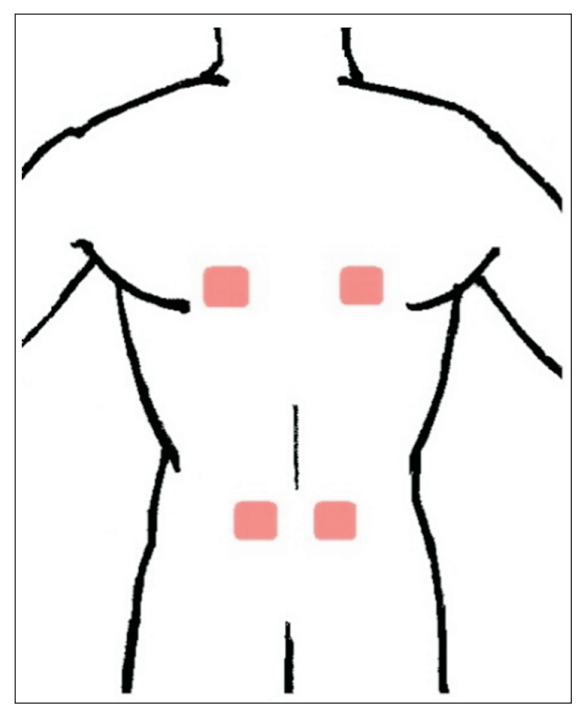

Fig. 3. Surface electrodes were attached to back muscles bilaterally at 4 site. Two $5 \mathrm{~cm}$ lateral to the T6 spinous process, the rest $2 \mathrm{~cm}$ lateral to the $\mathrm{L} 5$ spinous process. 

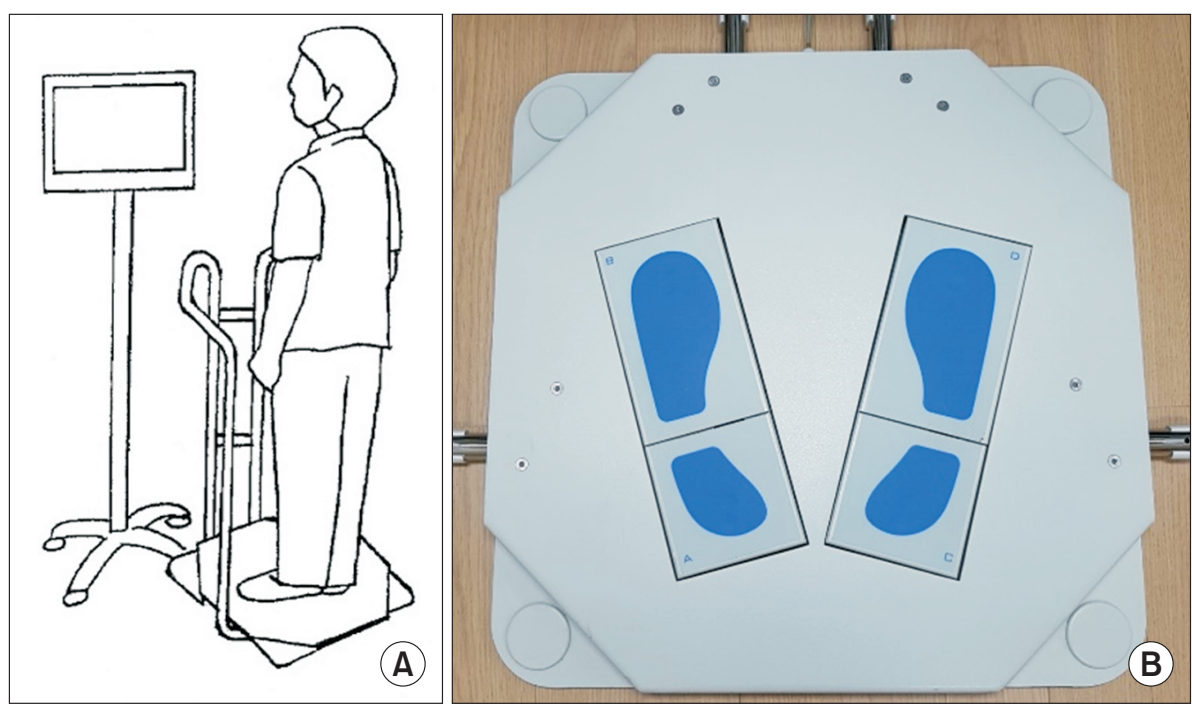

Fig. 4. (A) Static posturographic device (Tetrax). (B) Four reference points (bilateral toe and heel parts) were used to determine the pressure. problems, and the higher the score, the lower the stability. Each parameter was expressed by comparison with the mean, in units of standard deviation (SD). The normal range varies from $1.0 \mathrm{SD}$ below the mean to $1.5 \mathrm{SD}$ above.

\section{Statistical analysis}

The SPSS (Statistical Package of Social Science) version 14.0K for Windows (SPSS Inc., Chicago, IL, USA) was used to analyze data and the level of statistical significance was set to $\mathrm{p}<0.05$. For baseline characteristics, we used the Kruskal-Wallis test for continuous variables and Fisher exact test for categorical variables. The results were reported as a number on a categorical scale, and as the mean \pm SD on continuous scale. The Wilcoxon signedrank test was used to compare each group before and after treatment. The Kruskal-Wallis test was used to compare the effects of interventions among the three groups, and the Mann-Whitney U-test with the Bonferroni correction was used for post-hoc analysis. We set a level of statistical significance at $\mathrm{p}<0.05$ for the Kruskal-Wallis test, and $\mathrm{p}<0.017$ for the Mann Whitney U-test.

\section{RESULTS}

No significant differences were detected in demographic or clinical characteristics of subjects between the groups ( $p>0.05)$ (Table 1). All subjects completed the entire study without any side effects associated with intervention. However, since the post-urographic test us- ing Tetrax for secondary outcome was conducted in only those who achieved static standing without holding, 8 subjects in group A, 7 subjects in group B, and 8 subjects in group $\mathrm{C}$ were evaluated.

No significant differences were observed in the baseline outcomes among the three groups $(p>0.05)$. Comparison of each of the three groups before and after treatment revealed statistically significant changes in K-BBS, TIS, KMBI, WDI, and SI in all groups ( $\mathrm{p}<0.05)$ (Table 2$)$.

Improvements in BBS and TIS were significantly higher in groups A and B than in group C, whereas K-MBI and SI failed to show any significant difference. In the case of WDI, although not statistically significant, the possibility of group differences was observed ( $\mathrm{p}=0.051)$. Post-hoc analysis indicated a higher improvement in in K-BBS and TIS among groups A and B compared with group C. However, no significant difference in K-BBS, TIS, K-MBI, WDI, and SI was detected between groups A and B (Table 3).

\section{DISCUSSION}

Previous studies have focused on limb muscles for the treatment and prognosis of patients after stroke [12-14]. However, the importance of core muscles and the related rehabilitation therapies have recently been published $[1,3,15-17]$. Core muscles include the diaphragm as the roof, pelvic floor muscles as base, the abdominal muscles in front, and the paraspinalis muscles in the back [1]. These muscles stabilize the spine, and provide a stable support for the limbs during various activities. Core sta- 
Table 1. Demographics and baseline clinical characteristics

\begin{tabular}{|c|c|c|c|c|}
\hline Variable & Group A $(n=10)$ & Group B $(n=10)$ & Group C $(n=10)$ & p-value ${ }^{a)}$ \\
\hline Age (yr) & $59.4 \pm 11.74$ & $68.6 \pm 13.57$ & $57.5 \pm 11.84$ & 0.121 \\
\hline \multicolumn{5}{|l|}{ Sex } \\
\hline Male & $6(60)$ & $6(60)$ & $9(90)$ & 0.297 \\
\hline Female & $4(40)$ & $4(40)$ & $1(10)$ & \\
\hline \multicolumn{5}{|l|}{ Hemiside } \\
\hline Right & $4(40)$ & $2(20)$ & $6(60)$ & 0.248 \\
\hline Left & $6(60)$ & $8(80)$ & $4(40)$ & \\
\hline \multicolumn{5}{|l|}{ Etiology } \\
\hline Infarction & $7(70)$ & $6(60)$ & $7(70)$ & $>0.999$ \\
\hline Hemorrhage & $3(30)$ & $4(40)$ & $3(30)$ & \\
\hline Time after stroke (day) & $16.5 \pm 8.18$ & $17.3 \pm 9.12$ & $13.6 \pm 5.52$ & 0.540 \\
\hline K-MMSE & $23.3 \pm 8.67$ & $23.3 \pm 5.93$ & $22.2 \pm 8.77$ & 0.938 \\
\hline
\end{tabular}

Values are presented as mean \pm standard deviation or number (\%).

Group A, CME with NMES to abdominal muscles; Group B, CME with NMES to back muscles; Group C, CME alone; CME, core muscle strengthening exercise; NMES, neuromuscular electrical stimulation; K-MMSE, Korean version of Mini-Mental State Examination.

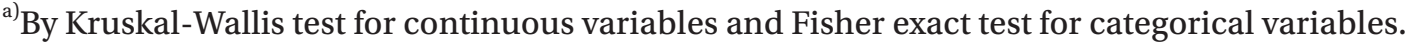

Table 2. Pre- and post-treatment comparison of the three groups

\begin{tabular}{|c|c|c|c|c|c|c|}
\hline & \multicolumn{2}{|c|}{ Group A } & \multicolumn{2}{|c|}{ Group B } & \multicolumn{2}{|c|}{ Group C } \\
\hline & Pre & Post & Pre & Post & Pre & Post \\
\hline K-BBS & $27.2 \pm 13.73$ & $45.7 \pm 7.26^{*}$ & $26.4 \pm 14.43$ & $46.3 \pm 8.82^{*}$ & $27.9 \pm 16.71$ & $36.3 \pm 13.30 *$ \\
\hline TIS & $12.0 \pm 3.40$ & $18.6 \pm 2.22^{*}$ & $12.4 \pm 4.01$ & $19.4 \pm 2.84^{*}$ & $12.8 \pm 5.03$ & $15.9 \pm 4.58^{*}$ \\
\hline K-MBI & $48.8 \pm 13.02$ & $76.7 \pm 11.50^{*}$ & $42.8 \pm 19.45$ & $75.3 \pm 15.19 *$ & $45.6 \pm 14.15$ & $70.2 \pm 12.00 *$ \\
\hline WDI & $9.1 \pm 3.19$ & $5.3 \pm 2.56^{*}$ & $10.18 \pm 3.70$ & $6.35 \pm 2.34^{*}$ & $9.42 \pm 5.67$ & $7.56 \pm 4.23^{*}$ \\
\hline SI & $46.8 \pm 21.67$ & $33.9 \pm 14.58^{*}$ & $44.7 \pm 19.31$ & $31.0 \pm 15.05^{*}$ & $48.3 \pm 16.86$ & $38.4 \pm 14.71^{*}$ \\
\hline
\end{tabular}

Values are presented as mean \pm standard deviation.

Group A, CME with NMES to abdominal muscles; Group B, CME with NMES to back muscles; Group C, CME alone; $\mathrm{CME}$, core muscle strengthening exercise; NMES, neuromuscular electrical stimulation; K-BBS, Korean version of Berg Balance Scale; TIS, Trunk Impairment Scale; K-MBI, Korean version of Modified Barthel Index; WDI, Weight Distribution Index; SI, Stability Index.

${ }^{*} \mathrm{p}<0.05$, by Wilcoxon signed-rank sum test for continuous variables.

bility plays a major role in maximizing the function and is a reliable predictor of patient recovery after stroke [8]. The results of this study were consistent with previous findings suggesting that core muscle strengthening improves the balance or ADL of post-stroke hemiplegic patients [4,6-10].

NMES is a technique in which muscle contraction is electrically stimulated in the area where surface electrodes are attached. It ameliorates muscle atrophy and weakness secondary to immobilization by preventing the decline in muscle protein synthesis, and improves the muscle strength via somatosensory stimulation that increases cortical excitability $[18,19]$. Several studies have shown that NMES combined with core strengthening exercise yields better rehabilitative effects than NMES or strengthening exercise alone $[6,20,21]$.

In most of the previous studies [6,10], NMES was applied only to paraspinalis muscles. However, this study showed no significant differences between groups A and $B$ in all outcome measures. NMES is contraindicated in patients with pressure ulcers, which are not an uncommon complication after stroke and occur mostly in the 
Table 3. Comparison of outcomes among the three groups

\begin{tabular}{|c|c|c|c|c|c|c|c|}
\hline \multirow{3}{*}{ K-BBS } & \multicolumn{3}{|c|}{$\Delta$ (post-pre) } & \multicolumn{4}{|c|}{ p-value } \\
\hline & \multirow{2}{*}{\begin{tabular}{|c|} 
Group A \\
$18.5 \pm 8.10$
\end{tabular}} & \multirow{2}{*}{$\begin{array}{c}\text { Group B } \\
19.9 \pm 5.44\end{array}$} & \multirow{2}{*}{$\begin{array}{c}\text { Group C } \\
8.4 \pm 4.14\end{array}$} & \multirow{2}{*}{$\begin{array}{c}\text { Across } 3 \text { groups } \\
0.001^{\mathrm{a})}\end{array}$} & \multicolumn{3}{|c|}{ Between 2 groups } \\
\hline & & & & & A vs B & $A$ vs $C^{b)}$ & 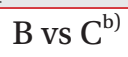 \\
\hline TIS & $6.6 \pm 1.90$ & $7.0 \pm 2.26$ & $3.1 \pm 0.99$ & $0.001^{a)}$ & A vs $B$ & $A$ vs $C^{b)}$ & $B$ vs $C^{b)}$ \\
\hline K-MBI & $28.1 \pm 8.02$ & $32.5 \pm 12.72$ & $24.6 \pm 10.56$ & 0.335 & A vs $B$ & A vs $\mathrm{C}$ & B vs $\mathrm{C}$ \\
\hline WDI & $-3.7 \pm 1.07$ & $-3.8 \pm 1.66$ & $-1.6 \pm 1.65$ & 0.051 & A vs $B$ & A vs $C$ & B vs $C$ \\
\hline SI & $-12.8 \pm 8.50$ & $-13.6 \pm 4.69$ & $-9.2 \pm 3.16$ & 0.252 & A vs B & A vs $C$ & B vs C \\
\hline
\end{tabular}

Values are presented as mean \pm standard deviation.

Group A, CME with NMES to abdominal muscles; Group B, CME with NMES to back muscles; Group C, CME alone; CME, core muscle strengthening exercise; NMES, neuromuscular electrical stimulation; K-BBS, Korean version of Berg Balance Scale; TIS, Trunk Impairment Scale; K-MBI, Korean version of Modified Barthel Index; WDI, Weight Distribution Index; SI, Stability Index.

${ }^{a)} \mathrm{p}<0.05,{ }^{\text {b) }} \mathrm{p}<0.017$, by Kruskal-Wallis test for continuous variables and Mann-Whitney U-test for post-hoc analysis.

back [22]. Patients with postoperative wounds and scars in the back area may also have limited application of NMES to the back muscles. Therefore, NMES to the abdominal muscles may be an alternative in post-stroke hemiplegic patients for improving postural balance.

NMES may have additional benefits on the abdominal muscles. Kang et al. [23] reported that abdominal muscle strengthening is effective in improving pulmonary function by increasing abdominal cavity pressure and expiratory capacity. In addition, NMES to abdominal muscles may reduce low back pain by strengthening the deep lumbar stabilizer muscles [11,24].

In this study, no significant difference was observed between groups A and B in overall outcomes, due to several possible factors. Core muscles induce trunk motion and increase stability by acting synergistically and contracting cooperatively. Further, each core muscle exhibits several functions depending on the situation. For example, the quadratus lumborum facilitates not only trunk extension but also lateral bending and even flexion [1]. Therefore, indirect NMES effects are observed on the overall core muscles in both cases of electrical stimulation: abdominal and back muscles. Additionally, because core muscles consist of several large and small, and superficial and deep muscles, it is almost impossible to selectively stimulate only the targeted muscle by NMES $[1,11]$. Therefore, although electrodes were attached to the thoracic erector spinae and lumbar erector spinae on back muscles, and to the transverse abdominis, obliquus internus and externus on abdominal muscles [6,11], NMES might affect other core muscles as well as the targeted muscles.
Interestingly, the group B showed higher values than group A in all outcomes. Tanaka et al. [16] reported that the muscle strength of trunk extension in post-stroke hemiplegic patients decreased more than in flexion. This finding suggests that strengthening the back muscle as trunk extensor was more helpful than the abdominal muscle as trunk flexor, and was consistent with our study findings. Further research is needed to investigate whether NMES to the back and abdominal muscles together with CME improves postural balance more than NMES targeted at each muscle individually in post-stroke hemiplegic patients.

A significant difference was found in the TIS as well as the K-BBS between groups B and C. This result was inconsistent with that of a previous study [6], which showed a significant difference between the two groups in the dynamic sitting balance subscale of the TIS, but not in the total score of the TIS. As the reason of this, the first is that total amount of intervention between the two studies was different. In the previous study, intervention was performed three times weekly for 20 minutes daily over a period of 3 weeks, although we originally planned an intervention time as $\mathbf{3 0}$ minutes daily, 5 days per week for 3 weeks. For the second reason, the K-BBS, TIS, and K-MBI scores of the subjects participating in our study were higher than in the study of Ko et al. [6] suggesting that our participants exhibited better postural stability and function than in the previous study, and these differences in baseline characteristics might have affected the results. However, it was consistent with previous studies that CME with NMES on back muscles was better than 
CME alone in improving the balance of the post-stroke hemiplegic patients.

When comparing the improvement of K-MBI among the three groups, no significant differences were found in our study. As reported in a previous study [6], this finding may be attributed to the short follow-up period.

WDI and SI measured by posturographic test using Tetrax did not show a significant difference among the three groups compared with BBS and TIS. Textrax evaluates the postural stability and has been used to improve the balance abilities in various patient populations including post-stroke patients by assessing the interaction between the pressure of four reference points [25-29]. This tool is conducted only in the standing state and is sensitive to variables such as fatigue and sleeplessness [30], which may influence these results. However, interestingly, the comparison of improvement in WDI among the three groups showed a possibility of group differences $(p=0.051)$. A longer follow-up period and a larger sample size may yield a different outcome.

This study has the following limitations: first, due to the small number of subjects enrolled, it is difficult to generalize our results to post-stroke hemiplegic patients. Second, patients with relatively good function were enrolled. Third, since we followed up patients for only 3 weeks, the long-term effectiveness could not be determined. Fourth, as reported in other similar studies before, we could not control all the variables such as individual limb muscle strength, intensity of CME program and location of stroke that may affect the details of intervention or the results. Although we tried to reduce bias by performing all CME programs under the guidance of the physical therapist, a few patients failed to perform specific exercises due to their functional limitations and conditions. Finally, among three groups, there would be discrepancies in the degree of muscle activation as well as the intensity of NMES stimulation, because of differences in individual characteristics such as amounts of fat tissue and the motor threshold. In further studies, real time ultrasound imaging or surface electromyography would be required to identify the degree of muscle activation.

In conclusion, this is the first study that investigated the effects of NMES to abdominal muscles in postural balance, compared with those observed on the back muscles in post-stroke hemiplegic patients. Compared with CME alone, NMES to the abdominal muscles with CME is a more effective approach to rehabilitation. It also provides an alternative intervention in post-stroke hemiplegic patients contraindicated for NMES to the back muscles for improving postural balance. Further studies investigating the synergistic effects of NMES to the back and abdominal muscles are needed, adjusting the limitations of this study.

\section{CONFLICT OF INTEREST}

No potential conflict of interest relevant to this article was reported.

\section{ACKNOWLEDGMENTS}

This work was supported by the Soonchunhyang University Research Fund.

\section{REFERENCES}

1. Kibler WB, Press J, Sciascia A. The role of core stability in athletic function. Sports Med 2006;36:189-98.

2. Chuter VH, Janse de Jonge XA. Proximal and distal contributions to lower extremity injury: a review of the literature. Gait Posture 2012;36:7-15.

3. Hodges PW. Core stability exercise in chronic low back pain. Orthop Clin North Am 2003;34:245-54.

4. Karatas M, Cetin N, Bayramoglu M, Dilek A. Trunk muscle strength in relation to balance and functional disability in unihemispheric stroke patients. Am J Phys Med Rehabil 2004;83:81-7.

5. Merkert J, Butz S, Nieczaj R, Steinhagen-Thiessen E, Eckardt R. Combined whole body vibration and balance training using Vibrosphere: improvement of trunk stability, muscle tone, and postural control in stroke patients during early geriatric rehabilitation. $\mathrm{Z}$ Gerontol Geriatr 2011;44:256-61.

6. Ko EJ, Chun MH, Kim DY, Yi JH, Kim W, Hong J. The additive effects of core muscle strengthening and trunk NMES on trunk balance in stroke patients. Ann Rehabil Med 2016;40:142-51.

7. Yoo SD, Jeong YS, Kim DH, Lee M, Noh SG, Shin YW, et al. The efficacy of core strengthening on the trunk balance in patients with subacute stroke. J Korean Acad Rehabil Med 2010;34:677-82.

8. Yu SH, Park SD. The effects of core stability strength 
exercise on muscle activity and trunk impairment scale in stroke patients. J Exerc Rehabil 2013;9:362-7.

9. Fujita T, Sato A, Togashi Y, Kasahara R, Ohashi T, Yamamoto Y. Contribution of abdominal muscle strength to various activities of daily living of stroke patients with mild paralysis. J Phys Ther Sci 2015;27:815-8.

10. Kim YM, Chun MH, Kang SH, Ahn WH. The effect of neuromuscular electrical stimulation on trunk control in hemiparetic stroke patients. J Korean Acad Rehabil Med 2009;33:265-70.

11. Baek SO, Cho HK, Jung GS, Son SM, Cho YW, Ahn SH. Verification of an optimized stimulation point on the abdominal wall for transcutaneous neuromuscular electrical stimulation for activation of deep lumbar stabilizing muscles. Spine J 2014;14:2178-83.

12. Smith MC, Byblow WD, Barber PA, Stinear CM. Proportional recovery from lower limb motor impairment after stroke. Stroke 2017;48:1400-3.

13. Stinear CM, Byblow WD, Ackerley SJ, Barber PA, Smith MC. Predicting recovery potential for individual stroke patients increases rehabilitation efficiency. Stroke 2017;48:1011-9.

14. Cortes JC, Goldsmith J, Harran MD, Xu J, Kim N, Schambra HM, et al. A short and distinct time window for recovery of arm motor control early after stroke revealed with a global measure of trajectory kinematics. Neurorehabil Neural Repair 2017;31:552-60.

15. Bohannon RW. Lateral trunk flexion strength: impairment, measurement reliability and implications following unilateral brain lesion. Int J Rehabil Res 1992;15:249-51.

16. Tanaka S, Hachisuka K, Ogata H. Muscle strength of trunk flexion-extension in post-stroke hemiplegic patients. Am J Phys Med Rehabil 1998;77:288-90.

17. Vieira S, Dibai-Filho AV, Brandino HE, Ferreira VT, Scheicher ME. Abdominal muscle strength is related to the quality of life among older adults with lumbar osteoarthritis. J Bodyw Mov Ther 2015;19:273-7.

18. Gibson JN, Smith K, Rennie MJ. Prevention of disuse muscle atrophy by means of electrical stimulation: maintenance of protein synthesis. Lancet 1988;2:767-70.

19. Maffiuletti NA, Roig M, Karatzanos E, Nanas S. Neuromuscular electrical stimulation for preventing skeletal-muscle weakness and wasting in critically ill patients: a systematic review. BMC Med 2013;11:137.
20. Qi YC, Niu XL, Gao YR, Wang HB, Hu M, Dong LP, et al. Therapeutic effect evaluation of neuromuscular electrical stimulation with or without strengthening exercise on spastic cerebral palsy. Clin Pediatr (Phila) 2018;57:580-3.

21. Alon G, McCombe SA, Koutsantonis S, Stumphauzer LJ, Burgwin KC, Parent MM, et al. Comparison of the effects of electrical stimulation and exercise on abdominal musculature. J Orthop Sports Phys Ther 1987;8:567-73.

22. Frontera WR, DeLisa JA. DeLisa's physical medicine and rehabilitation. 5th ed. Philadelphia: Lippincott Williams and Wilkins; 2010. p. 1393-409.

23. Kang JI, Kim BR, Park SK, Yang DJ, Jeong DK, Kim JH. Effects of deep abdominal muscle strengthening exercises on pulmonary function and the ability to balance in stroke patients. J Korea Phys Ther 2015;27:25863.

24. Coghlan S, Crowe L, McCarthypersson U, Minogue C, Caulfield B. Neuromuscular electrical stimulation training results in enhanced activation of spinal stabilizing muscles during spinal loading and improvements in pain ratings. Conf Proc IEEE Eng Med Biol Soc 2011;2011:7622-5.

25. Akkaya N, Doganlar N, Celik E, Aysse SE, Akkaya S, Gungor HR, et al. Test-retest reliability of Tetrax static posturography system in young adults with low physical activity level. Int J Sports Phys Ther 2015;10:893-900.

26. Quatman-Yates CC, Lee A, Hugentobler JA, Kurowski BG, Myer GD, Riley MA. Test-retest consistency of a postural sway assessment protocol for adolescent athletes measured with a force plate. Int J Sports Phys Ther 2013;8:741-8.

27. Kohen-Raz R. Application of tetra-ataxiametric posturography in clinical and developmental diagnosis. Percept Mot Skills 1991;73:635-56.

28. Klavora P, Warren M. Rehabilitation of visuomotor skills in poststroke patients using the Dynavision apparatus. Percept Mot Skills 1998;86:23-30.

29. Lee NH, Lee J, Lee KN. The effects of treatment with a TETRAX on balance and mobility in acute stroke patients. Phys Ther Korea 2010;17:11-9.

30. Ma J, Yao YJ, Ma RM, Li JQ, Wang T, Li XJ, et al. Effects of sleep deprivation on human postural control, subjective fatigue assessment and psychomotor performance. J Int Med Res 2009;37:1311-20. 